\title{
Robotics for Healthcare Productivity Improvement
}

\author{
Yu Haoyong \\ Department of Biomedical Engineering, National University of Singapore \\ Singapore
}

The population aging has resulted in escalating cost in healthcare due to increasing number of patients and the decreasing number of healthcare workers. Robotics technologies offer great potential to reduce cost in healthcare by improving the productivity. Robots can be also be used to improve outcomes of treatment such as physical therapy for stroke patients.

In this talk, a broad background and significance of the rehab and assistive robotics will be given and the key research issues, such as actuator design, sensing, and control will be highlighted. Introduction of the development of rehabilitation robots for neurorehabilitation as well as assistive robots at Biorobotics Lab at the National University of Singapore will also be introduced, which include exoskeleton robots and smart walker for gait training and smart hospital bed movers for healthcare workers. 